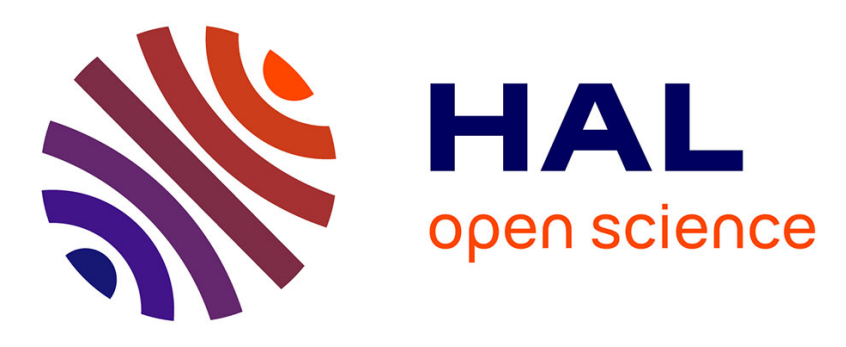

\title{
Reconfigurable Photonic Logic Architecture: An Overview
}

\author{
Vitor Silva, Manuel Barata, Manuela Vieira
}

\section{To cite this version:}

Vitor Silva, Manuel Barata, Manuela Vieira. Reconfigurable Photonic Logic Architecture: An Overview. 8th Doctoral Conference on Computing, Electrical and Industrial Systems (DoCEIS), May 2017, Costa de Caparica, Portugal. pp.447-462, 10.1007/978-3-319-56077-9_44 . hal-01629599

\section{HAL Id: hal-01629599 \\ https://hal.inria.fr/hal-01629599}

Submitted on 6 Nov 2017

HAL is a multi-disciplinary open access archive for the deposit and dissemination of scientific research documents, whether they are published or not. The documents may come from teaching and research institutions in France or abroad, or from public or private research centers.
L'archive ouverte pluridisciplinaire HAL, est destinée au dépôt et à la diffusion de documents scientifiques de niveau recherche, publiés ou non, émanant des établissements d'enseignement et de recherche français ou étrangers, des laboratoires publics ou privés. 


\title{
Reconfigurable Photonic Logic Architecture: An Overview
}

\author{
Vitor Silva $^{1,2}$, Manuel Barata ${ }^{1,2}$ and Manuela Vieira ${ }^{1,2,3}$. \\ ${ }^{1}$ Electronics Telecommunication and Computer Department - ISEL Instituto Superior de \\ Engenharia de Lisboa / IPL - Instituto Politécnico de Lisboa, 1959-007 Lisboa, Portugal \\ ${ }^{2}$ CTS-UNINOVA, Quinta da Torre, Monte Caparica, 2829-516, Caparica, Portugal. \\ ${ }^{3}$ DEE-FCT-UNL, Quinta da Torre, Monte Caparica, 2829-516, Caparica, Portugal \\ vsilva@deetc.isel.ipl.pt
}

\begin{abstract}
The photosensor studied in this document, is an amorphous silicon structure deposited on transparent glass allowing illumination on both sides. It responds to wavelengths from near infrared range to the ultra-violet. The front illumination surface is used for inputting light signal. The dynamic characteristics of the photosensor are altered by using optical bias on either surface of the sensor, thus the same input results in different outputs. Experimental studies made with the photosensor evaluate its applicability as a multiplexer, demultiplexer and logical operations device. A memory effect was observed. A programmable pattern emission system, and a validation and recovery system were built to illuminate the sensor with input light signals and to analyze the resulting output.
\end{abstract}

Keywords: Optoelectronics, photosensor, a-SiC technology, pin, amorphous silicon, logical functions, digital light signal.

\section{Introduction}

Research work of the a-Si:H, a-SiC:H pi'npin photosensor [1] has shown that it has a nonlinear property of changing its bandwidth not only with the electrical bias but also a change of its bandwidth occurs with a light shining over the sensor, in either surface, despite the applied electrical bias [2]. Several applications have been done or proposed and the possibility of using this sensor for communication is studied even though the throughput is presently $12000 \mathrm{bps}$ per channel.

This suggests that the channel selection which is made by digital switching over electrical channels to be de-multiplexed can, with this approach, be selected at the sensor with light selectors thus bringing to the photon side of the interface the capability of choosing the desired channel. Using Manchester code instead of the previous work with NRZ (No Return to Zero) code improves channel discrimination at the reception. The sensor is also looked upon its capability of producing a single electrical output with several input channels. This photocurrent translation is studied to identify logical operations as the AND, OR, NOT and XOR, and other more complex as the adder. A memory effect has also been identified and studied herein. Experiments with random values show the dispersion of the signals at the reception and

adfa, p. 441, 2011.

(C) Springer-Verlag Berlin Heidelberg 2011 
several strategies to reproduce the input channels are proposed. Several programs had to be built and are used as tools to illuminate the sensor and analyze the sensor output. Observation of the device's behavior arose several questions that span the proposed work: How many channels can be transmitted by WDM, in visible light, with the sensor by selecting the desired channel with ultra-violet light?; What wavelengths ranges are best suited for the de-multiplexing function?; Which are the logical functions that can be translated by this sensor?; How to build complex logical functions with the device?; How to de-multiplex light channels using light selectors with one sensor?; How to build a memory due to charges stored by light?. The answer to these questions is presented in this document, along with other study paths.

\section{Relationship to Smart System}

A smart system uses a feedback loop of data, providing evidence for decision-making. The system can monitor, measure, analyze, communicate and act, based on the sensors' information [3]. Smart systems can be classified in several levels: those that collect usage and performance data to be used for a next version of a smarter system, those that collect, process and present data for humans to make a better decision, and those that collect data to take action without any human intervention.

A smart system is based on data, and that is its core. Data can be easily acquired and stored but it will also easily increase in quantity. As a resource, its ownership is also a point to be taken into consideration. Data must be analyzed, fused with other data sources, interpreted and sourced into mathematical models that will hopefully provide a highly reliable decision making tool for society and especially for those whose jobs are involved in taking decisions.

A system needs feedback, and that aspect is fundamental in any smart system, not only to increase its performance but also to improve its robustness. Data of different sources may come from the same physical reality which brings our attention to redundancy. This redundancy, which can also be constituted by different systems, is also an advantage due to possible error conditions or flaws.

The device presented in this paper is a sensor that is sensitive to light and as such it can be inserted into a smart system data loop. One of the observed characteristics of the sensor is that it holds memory of the visible light that has impinged onto its surface, even when not connected to a circuit. This could be applied as a sensor to be located in a closed box or case; an environment where no electricity is present. If the box or case is opened under visible light and then shut again, it would be possible to know that at least one opening occurred by reading the sensor under a controlled no light condition.

\section{Device Structure and Operation}

The sensor is a two stacked pin structure (p(a-SiC:H)- i'(a-SiC:H)-n(a-SiC:H))-(p(a$\mathrm{SiC}: \mathrm{H})-\mathrm{i}(\mathrm{a}-\mathrm{Si}: \mathrm{H})-\mathrm{n}(\mathrm{a}-\mathrm{Si}: \mathrm{H}))$. Thicknesses and optical gap optimized for light absorption in the red and blue ranges; i- $(1000 \mathrm{~nm} ; 1.8 \mathrm{eV})$, i'- $(200 \mathrm{~nm} ; 2.1 \mathrm{eV})$ [4]. This 
structure is $1 \mathrm{~cm}^{2}$ in area, built with silicon carbon [5], can be seen in Fig. 1, where the wavelength arrows indicate absorption depths during operation. The wavelengths mostly used are: $\lambda_{V}=400 \mathrm{~nm}, \lambda_{B}=470 \mathrm{~nm}, \lambda_{\mathrm{G}}=524 \mathrm{~nm}$ and $\lambda_{\mathrm{R}}=626 \mathrm{~nm}$.

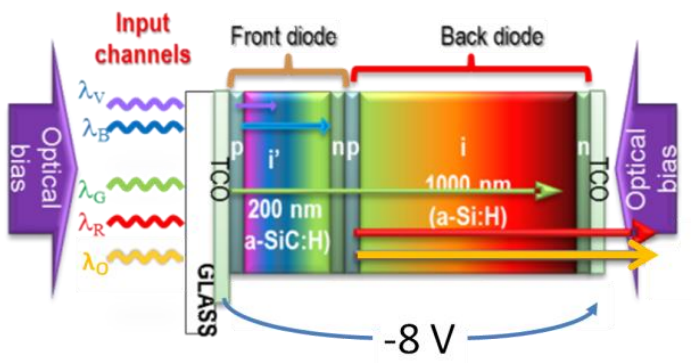

Fig. 1. Sensor structure and operation

The experimental setup use LEDs as light sources: as digital signals and as bias lighting. The digital signals are usually: violet $(400 \mathrm{~nm})$, blue $(470 \mathrm{~nm})$, green $(524$ $\mathrm{nm})$ and red $(626 \mathrm{~nm})$, and shone over the entire front surface of the device. The background lighting is ultra-violet $(390 \mathrm{~nm})$ and is set either at the back or at the front side of the sensor, and lighting is applied in a continuous non pulsating flux. Compared to the optical bias intensity, the intensity of the signal sources is lower.

Using a monochromator with $10 \mathrm{~nm}$ steps, from 300 to $800 \mathrm{~nm}$, spectral response curves were produced, and the resulting photocurrent stored in a file with the corresponding input wavelength. The device operation point was set at $-8 \mathrm{~V}$ because a previous study suggests that, that electrical bias is the most suited to allow for a steady state optical bias and channels with visible light impulses, while outputting a photocurrent that changes with the input wavelengths [6]. The spectral response for a violet $(400 \mathrm{~nm})$ optical bias at the front and back surfaces of the sensor is presented in Fig. 2.

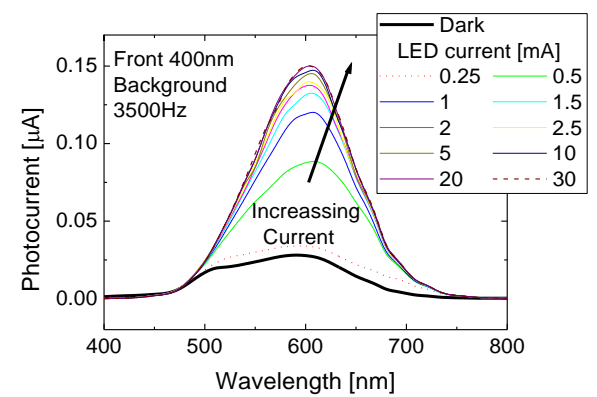

a)

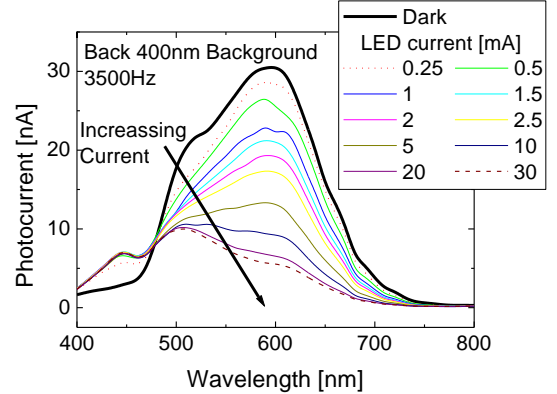

b)

Fig. 2. Sensor photocurrent output using optical bias wavelength of $400 \mathrm{~nm}$ at the a) front and b) back sides.

The experimental results of Fig. 2 a), show that the photocurrent increases in the 470 to $700 \mathrm{~nm}$ bandwidth when the sensor is lit on the front surface. To compare both graphs in of Fig. 2, the thick black curve represents the dark level and is the same on 
both figures. With increasing LED current through the LED that shines over the back surface, the photocurrent decreases gradually in the $470-700 \mathrm{~nm}$ bandwidths, and there is an almost identical increase of the photocurrent in the $400-470 \mathrm{~nm}$ range. The photocurrent gain, which is the output photocurrent divided by the dark current level, is shown in Fig. 3.
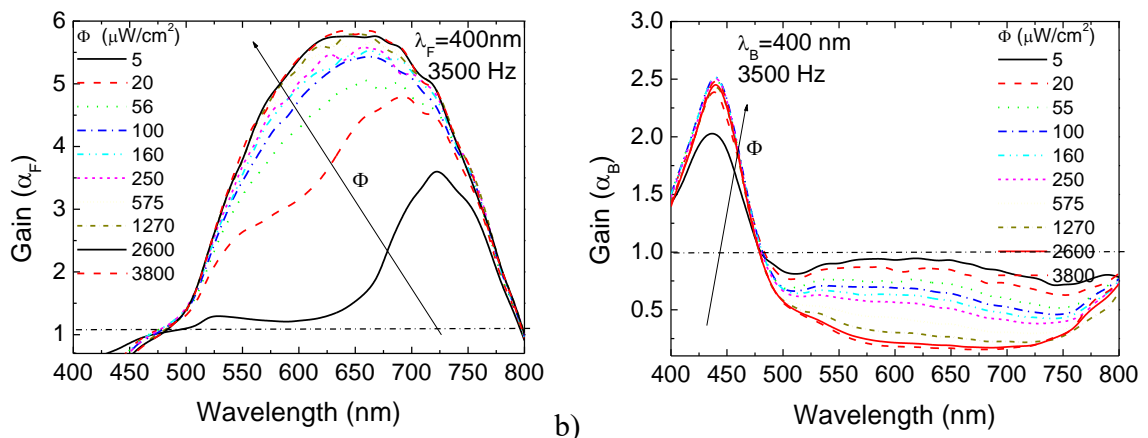

Fig. 3. Photocurrent gain due to bias lighting upon the a) front and b) back surfaces.

The spectral gain shown in Fig. 3a), is reduced within the short wavelengths $(<470$ $\mathrm{nm})$ and increased over the long wavelengths ( $>470 \mathrm{~nm}$ ), behaving as a selective filter centered in $650 \mathrm{~nm}$ with bias illumination at the front. With bias lighting shining over the back surface, Fig. 3b), the opposite behavior is noticed; the short wavelengths gain increases while the long wavelengths gain decreases. This resembles a filter centered at $440 \mathrm{~nm}$. The sensor acts as a selective filter, where the gain of the short and long pass wavelengths is controlled by the optical bias. The gains of both filters suffer almost no changes when the LED currents are above $10 \mathrm{~mA}$.

\section{Experimental Setup}

The sensor is biased with $-8 \mathrm{~V}$ and the experimental setup shown in Fig. 4. Both end surfaces are clear for lighting. The setup shows the LEDs relative position to the sensor. The optical bias LEDs, Front and Back, are both ultra-violet $(390 \mathrm{~nm})$. An optical bias lit LED has its illumination maintained at a fixed level. The current of each LED can be controlled in 127 steps from 0 to $30 \mathrm{~mA}$, and both LEDs are never lit simultaneously. The data channel LEDs are: Ultra-violet (U: $390 \mathrm{~nm}$ ), Violet (V: $400 \mathrm{~nm}$ ), Navy (N: $430 \mathrm{~nm})$, Cyan (C: $460 \mathrm{~nm})$, Blue (B: $470 \mathrm{~nm})$, Green (G: $524 \mathrm{~nm})$, Lime $(\mathrm{L}: 565 \mathrm{~nm})$, Orange (O: $605 \mathrm{~nm})$, Red (R: $626 \mathrm{~nm})$ and Magenta (M: $640 \mathrm{~nm})$. Each data channel can have its current changed from 0 to $30 \mathrm{~mA}$ in 127 steps, but the values used are usually 0 to $5 \mathrm{~mA}$ range with different synchronized patterns. 


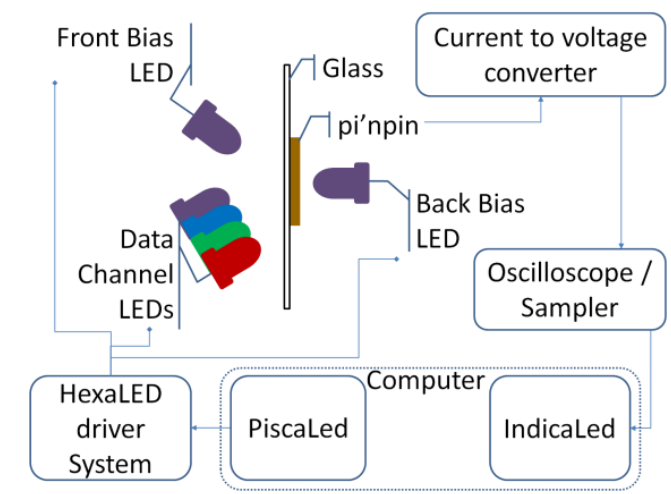

Fig. 4. Sensor and LED relative positions and equipment

All LEDs are software controlled by the PiscaLed [7, 8] system that drives them physically by communicating with the HexaLed driver [9]. Data values supplied by the PiscaLed are shaped into configurable frames and encoded or not in Manchester coding; the frame can be raw, with the data bits presented to the channel LEDs, in a frame with a preamble, start of frame, data values and end of frame. The preamble is a sequence of several [010101] bits on all channels, used at the reception to determine the maximum intensity value, used for normalization and synchronization. The start of frame begins with a slight difference in the preamble [0110] followed by data. The sequence of [010100] at the end of the frame precedes the switching off of all channels. To analyze the results several programs developed within this thesis were used namely the IndicaLed $[9,10,11]$.

Each data bit can be coded in non return to zero (NRZ) or Manchester [13]. When a large number of equal data bits are transmitted the resulting NRZ output is either no illumination ( $b i t s=0$ ) or continuous (bits $=1$ ); resulting in no photocurrent changes. With Manchester coding there is always a change in photocurrent due to the pulsed pattern. There are more solutions to overcome the NRZ pattern for example by the introduction of a scrambler or other type of encoding [14].

The photocurrent output of the sensor is named differently according to its optical illumination and signal source: dark, back or front. The Dark photocurrent is defined as the output current when the sensor is in darkness. The Front signal is the photocurrent output with optical bias applied to the front surface of the sensor and pulsing data LEDs shining, and consequently the Back photocurrent output is the electrical signal produced with the optical bias at the back surface and the pulsating data LEDs lit.

The photocurrent output is converted to a voltage by the current to voltage converter and is connected to an oscilloscope. The voltage signal is sampled at the oscilloscope and sent to the computer, as shown in Fig. 4. The Data stored consists on the data LEDs input and converted output photocurrent, so that the analysis can be validated by having both the expected result and the obtained output. The signals are stored as received, Dark, Front and Back signals read with their minimum value set to $0.0 \mathrm{~V}$, and normalized between 0.0 and 1.0 for analysis. 


\section{Five Channel WDM}

The multiplexing of several data channels into one channel is an efficient way to communicate between two peers with the demultiplexing of the single channel into the original ones. Wavelength division multiplexing (WDM) uses different wavelengths for each channel. A five channel WDM with each channel at $12000 \mathrm{bps}$ allows for a raw transmission rate of $5^{*} 12000=60000 \mathrm{bps}$. Two selectors, the front and back illumination of the background, are used to select the channels. As a summary the front illumination would sieve two channels and the back illumination would sieve another two, and the remaining channel selection process has a different approach [15]. To study the five channels multiplexer an experiment was made and the result is displayed in Fig. 5.

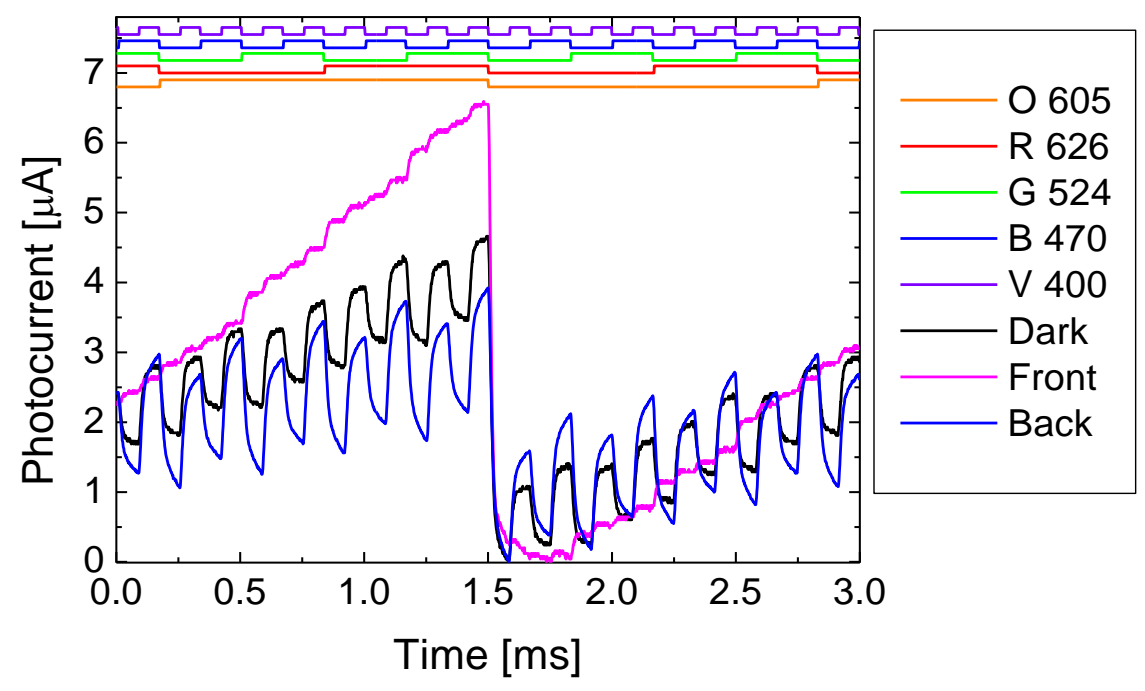

Fig. 5. A five channel multiplexer output.

Shown in Fig. 5 is the output of the pi'npin when it is bathed by the signal wavelengths (dark) and simultaneously by the front illumination of the background (front) and the background at the back of the device (back). The digital input signals applied to the LEDs, at the top of the figure, contain the input data to each channel. These digital signals help guiding the eyes to the front signal and showing that all 32 combinations are there. The back signal follows the violet input channel $(400 \mathrm{~nm})$ very strongly and distinguishes between dubious classifications of the front signal. The blue $(470 \mathrm{~nm})$ channel can also be recovered by the back signal. After the extraction of both violet and blue channels, the red $=626 \mathrm{~nm}$, orange $=605 \mathrm{~nm}$ and green $=524 \mathrm{~nm}$ can be easily identified.

The orange wavelength can be extracted because the gain of that wavelength $\left(\alpha_{\mathrm{O}-}\right.$ front $=4.53, \alpha_{\text {Oback }}=0.54$ ) is different from the other gains. Using the clustering approach [16], applied to the front and back signals of Fig. 5 results in what is shown in Fig. 6. 

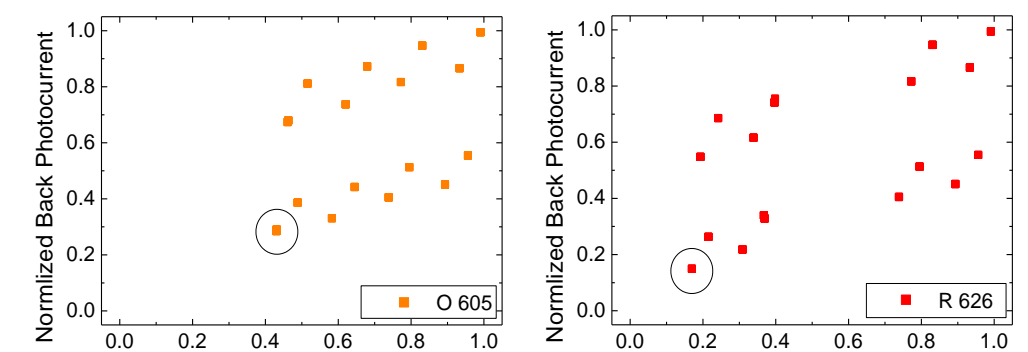

a)

b)

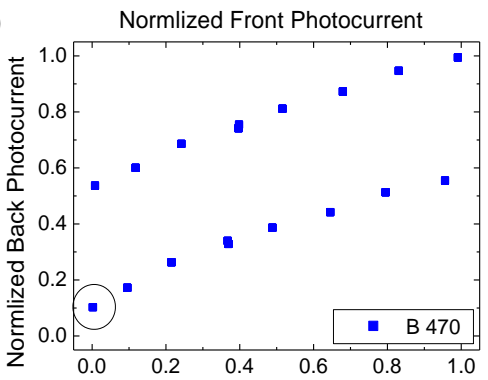

c)

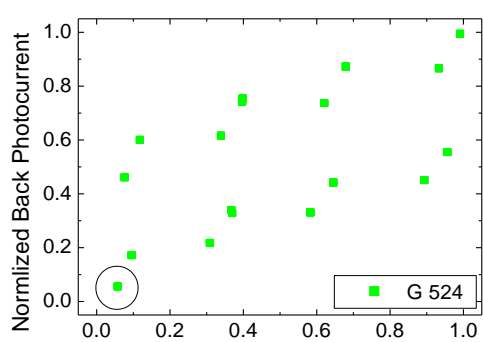

d)

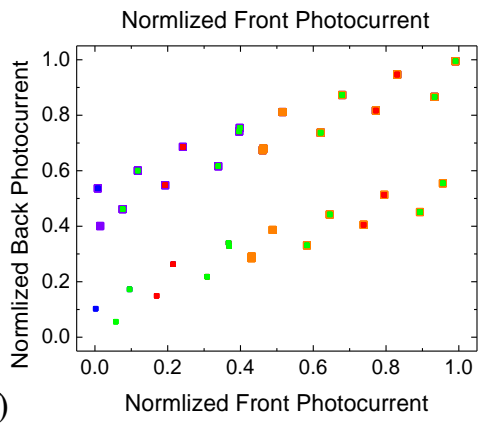

e)

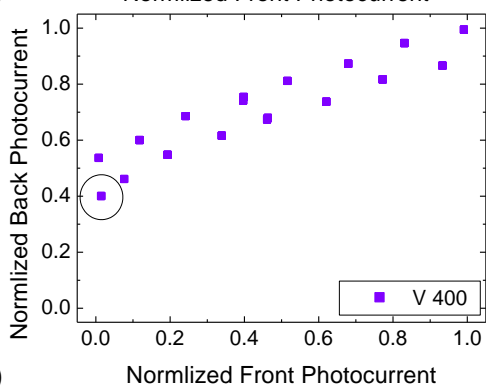

f)

Fig. 6. Clusters of a) $\mathrm{O}=605 \mathrm{~nm} \mathrm{~b}) \mathrm{R}=626 \mathrm{~nm} \mathrm{c}$ ) $\mathrm{G}=524 \mathrm{~nm} \mathrm{~d}) \mathrm{B}=470 \mathrm{~nm}$ e) $\mathrm{V}=400 \mathrm{~nm}$ and f) all ORGBV.

The pi'npin sensor responds to the orange O $605 \mathrm{~nm}$ signal depending on the other signals that are also shining at the sensor. With five different wavelengths, $2^{5}=32$ possible combinations lead to the 16 areas in Fig. 6 a) which represent the combinations that have the presence of the $\mathrm{O} 605$ wavelength. The area marked with a circle belongs to the influence of $\mathrm{O} 605$ when no other signal wavelength is active. The clusters for the other wavelengths are also shown in Fig. 6 b) to e). The graph of Fig. $6 \mathrm{f}$ ) is the over position of all individual wavelengths. There are only 31 areas on this graph because de 00000 bit is not plotted, as each individual graph only indicates where the wavelength is active.

Additional channels may be introduced using only the front and back selectors, but due to the cross gains of one channel over another difficult the extraction of the individual channels [16]. 


\section{Logic Functions}

The logic functions that are the basic of present digital technologies are mathematic operators of Boolean Algebra. The theoretical work done by George Boole in 1847 is presented in "The mathematical analysis of Logic" [17]. Using the Boolean logical functions with mathematical state theory more complex structures were built creating the reliable computers that are available at this date [18].

The Boolean logic functions, based in mathematical set theory, necessary to build other logic functions are: NOT (negation), AND (conjunction), OR (disjunction) [19]. The implementation of these mathematical constructs in hardware has been done with several techniques [20]. The basic logical functions can be implemented on a pi'npin device [21], as well as the Majority function, which is also known as the voting function [22].

\subsection{Digital Light Signals}

Logical functions are used as logic gates [23] and other logical functions are created by combining those basic gates. Some circuits are known by a name due to their function. One of those circuits is the multiplexer which is a combinatorial function. The multiplexer can be used as a basic circuit producing results as simple as the basic logic gates. The NOT function is one of those applications built with a multiplexer and is presented in Fig. 7 [24].

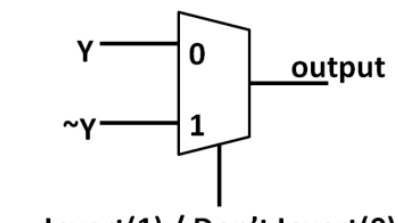

a) Inv

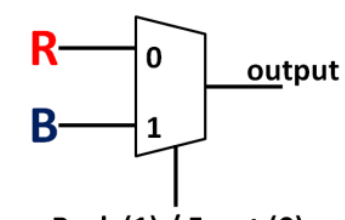

b)
Back (1) / Front (0)

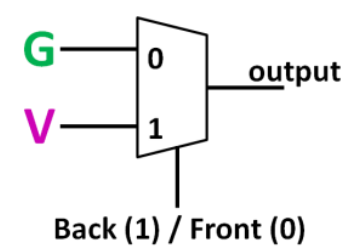

Fig. 7. A multiplexer is used a) as an invert function, and b) 2 examples of the NOT function using the digital light signal pair (Red, Blue) and (Green, Violet) pair.

The 2x1 multiplexer shown in Fig. 7 a) composed by a selector that chooses inputs $\mathrm{Y}$ and $\sim \mathrm{Y}$ (NOT Y). When Invert input is 1 , the output follows $\mathrm{Y}$, and when the Invert selector is 0 the output follows $\sim$ Y. The Invert selector chooses between: a $Y$ signal, or its inverse $\sim$ Y. Using the sensor's multiplexer capability [25] to act as an inverter is the basis of the work. Equation 1 shows, circuit in Fig. 7a), the following transformations:

output $=Y \cdot \sim$ Invert $+\sim Y \cdot$ Invert $=Y \oplus$ Invert

Equation 1, simplified as the exclusive OR (XOR) relation between Y and Invert inputs. If Invert is 0 the output follows Y input, otherwise it follows $\sim$ Y [26].

A digital light signal is defined as: a signal pair with two components where one is the inverse of the other, and the wavelengths of one is from the long and the other from the short filter ranges [27]. 
Any long, short combination as a signal pair can be used, for example: (Red, Blue) pair and (Green, Violet), both shown in Fig. 7 b).

The two different digital light signal pairs are presented in Fig. 8 with the same data sequence.
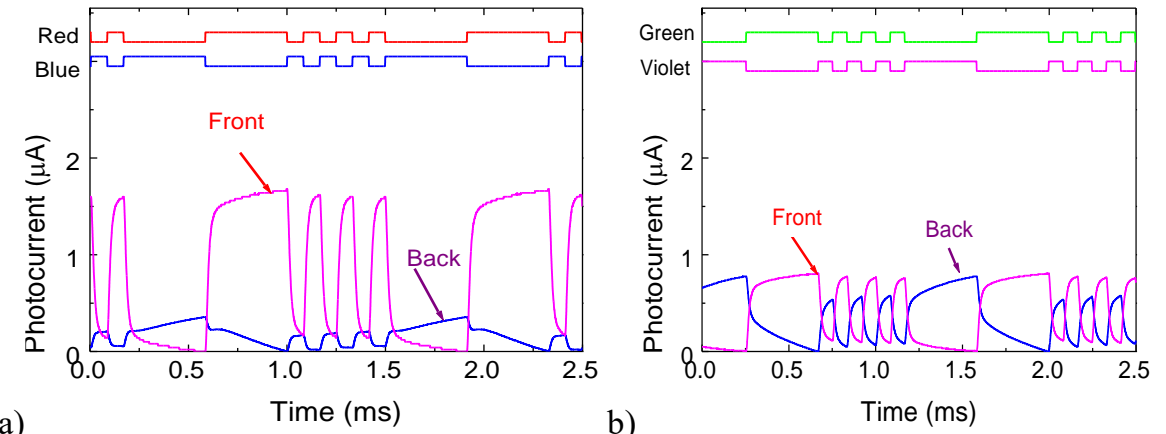

Fig. 8. Examples of digital light signals: a) Red-Blue pair, b) Green-Violet pair.

By subjecting both digital light signal pairs shown in Fig. 8 to the Front and Back bias lighting, it is clear that the Front signal follows the Red and Green input, and Back signal follows Blue and Violet signals.

A visible digital light signal $\mathrm{D}$ is the multiplexed signal of two wavelength channels; from the long and from the short wavelength ranges. Represented as D[L, S] where L and S represent the channel color [26], long and short-wavelength range, respectively.

\section{NOT Gate.}

The NOT gate shown in Fig. 7 b) is built by a long and a short wavelength channels (for example red and blue or green and violet) is controlled by the violet irradiation. The construction of this gate urged the definition of the digital light signal. Thus the digital light signal is the base of all logical functions built with this device. Fig. 8 shows examples of the NOT function, with the Back ultra-violet light.

Transmitting a False or a True value for a long period of time would degrade the recovery of the output signals, as only the ac component is used. To improve the photocurrent output it is necessary that a True or False bit value changes in time during its bit length. This can be solved by using the Manchester coding [13] which guarantees that there is a polarity change in the middle of each True or False bit time length. The photocurrent to be useful must be time dependent, so the logic signal, even if does not have its state changed, must vary in time. This obliges the digital signals to be synchronized and differential, thus a digital light signal must be Manchester coded $[20,21]$.

Two examples of digital light signals with Manchester coding are shown in Fig. 9. 


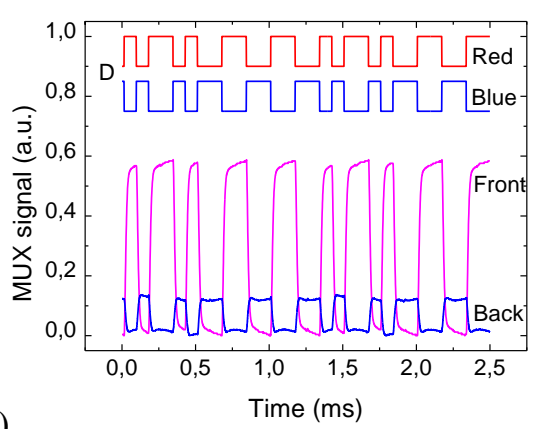

a)

Fig. 9. Digital light signals and its multiplexer output signals under front and back irradiation a) $\mathrm{D}$ (Red, Blue) and b) P(Green, Violet)

Two digital light signals are show in Fig. 9: (a) D[R, B] and (b) $P[G, V]$. By changing the background side illumination with an appropriate intensity and wavelength, results show that the digital light signal describes the basic logic functions [26]. Digital light signals $D[R, B]$ and $P[G, V]$ are plotted as a scatter graph in Fig. 10.
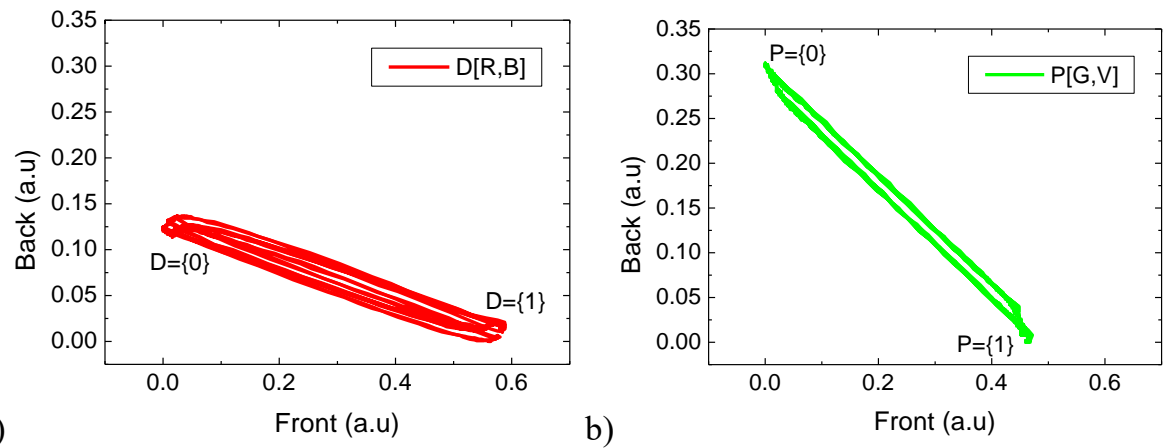

Fig. 10. Scatter graphs of Fig. 8 a) D[R, B] and b) $P[G, V]$

Shown in Fig. 10 are the scatter graphs of the signals presented in Fig. 9. It is clear that only two values exist in each digital light signal $\mathrm{D}[\mathrm{R}, \mathrm{B}]=\{\{0\},\{1\}\}$, and $\mathrm{P}[\mathrm{G}$, $\mathrm{V}]=\{\{0\},\{1\}\}$. The scatter graph also shows that the front and back signal are inversely correlated, due to the negative slope of the graphs [28] as expected due to each digital light signal being a composition of a short and long wavelength [29].

A digital light signal can be written as Equation 1.

$$
D[R, B]=\left[\begin{array}{ll}
R(t) \cdot i_{R} & B(t) \cdot i_{B}
\end{array}\right] \times\left[\begin{array}{ll}
\alpha_{\mathrm{R}, \text { Front }}^{\mathrm{V}} & \alpha_{\mathrm{R}, \text { Back }}^{\mathrm{V}} \\
\alpha_{\mathrm{B}, \text { Front }}^{\mathrm{V}} & \alpha_{\mathrm{B}, \text { Back }}^{\mathrm{V}}
\end{array}\right]=\left[\begin{array}{ll}
L_{R}(t) & S_{B}(t)
\end{array}\right]
$$

$R(t)$ is the digital sequence for example $=\{0,1,1,0,0,1,0,1\}$ and $B(t)$ the inverse of that sequence e.g. $=\{1,0,0,1,1,0,1,0\}$. The dark photocurrent for each wavelength is $i_{R}$ for the Red and $\mathrm{i}_{\mathrm{B}}$ for the Blue. 


\subsection{Interaction of Two Digital Light Signals}

Logic operators besides the NOT have expressions with two variables. Boolean logic has the associative and commutative properties of algebra that are applied when more than two variables are involved. The number of input variables and the operation that produces a single logical output is called a gate. In this section two light variables are studied as the basic building block of a logical gate. In order to effectively classify the output of the logical gate the sampling of the signal will also be addressed.

Two different digital light signals $\mathrm{D}[\mathrm{R}, \mathrm{B}]$ (Fig. 9a) and $\mathrm{P}[\mathrm{G}, \mathrm{V}]$ (Fig. 9b) illuminated the device and their combination is presented in Fig. 11 [27].

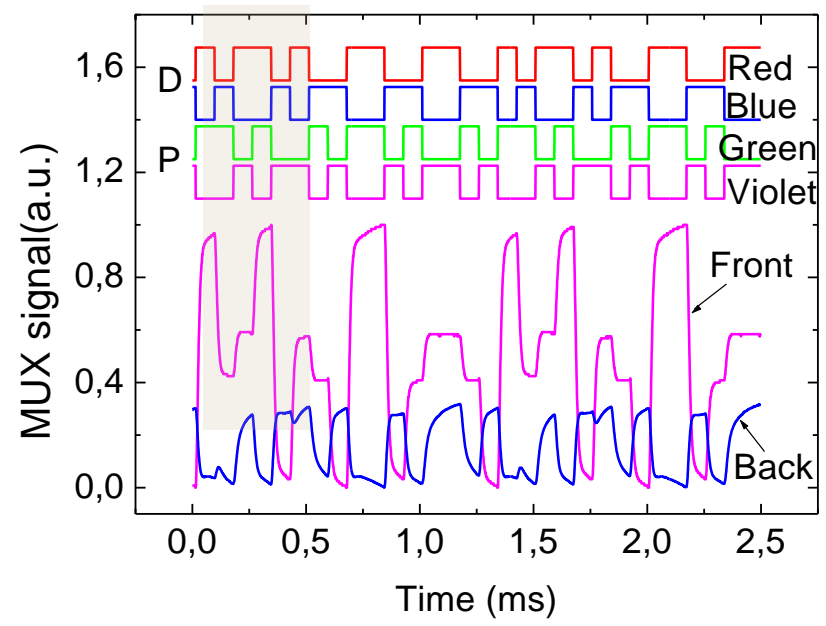

Fig. 11. Result of the interaction of two digital light signals D[Red, Blue] and $P[$ Green, Violet $]$

The interaction of two digital light signal pairs red-blue and green-violet is shown in Fig. 11. This signal is shown in Fig. 12 a) as a scatter graph where the Front signal is set as the horizontal axis and the Back signal is set as the vertical axis.

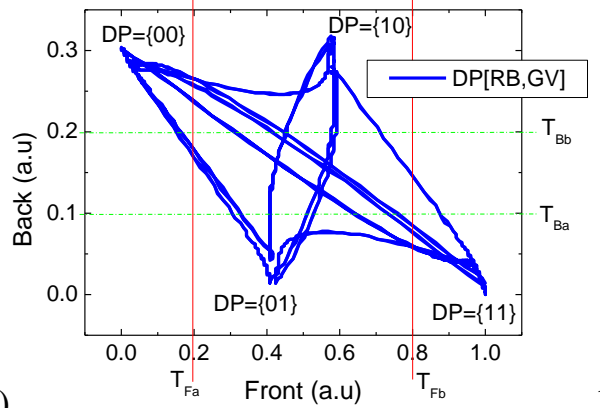

a)

Fig. 12. a) Scatter graph of the resulting Back and Front bias signals of Fig. 11 and b) detail of Fig. 11, contains all 4 combinations of two digital signals, front and back are the interaction of two signals D,P, and the OR, AND, XOR signals represent the expected output.

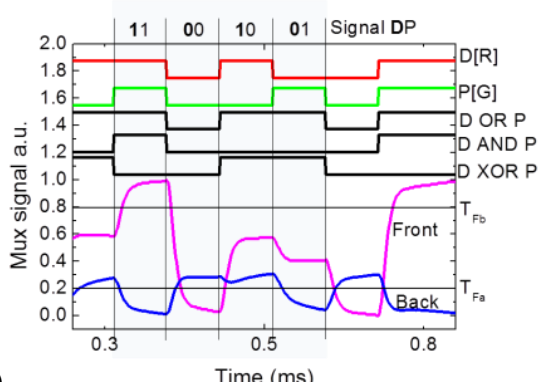

b) of 
The pattern selected in Fig. 11, shown in detail in Fig. 12 b), holds all possible combinations of the D and P signal and will be analyzed further in the text.

Fig. 12 shows signals Front and Back as a scatter graph, the sequence path where at each point, the back and front values meet at the same time instant i.e. function $O f($ Front $(t), B a c k(t))$. On the same figure the vertexes indicate the four combinations $\{00\},\{01\},\{10\}$ and $\{11\}$ of D and P. A simple classification grid can be used with threshold values which are drawn in solid and dashed lines on Fig. 12 a), to classify the logical output.

\section{AND, OR, XOR Gate.}

The selected logical function is built by choosing the threshold lines that define the result of the function.

D AND P logical function is defined as: True as output if and only if both inputs have True as a value i.e. $\{11\}$. In Fig. 11 corresponds to both Red and Green channels in their ON state (front highest level). Observing Fig. 11 and the scatter function of Fig. 12 a), a threshold line $\left(\mathrm{T}_{\mathrm{Fb}}\right)$ can be drawn above which, any result represents the output function D AND P, isolating the $\{11\}$ result.

The (D OR P) logical function is defined as having the output as True if either or both $\{\mathrm{DP}\}$ are True or equivalently the output is False if and only if both $\{\mathrm{DP}\}$ are False, i.e. $\{00\}$. Taking into account Fig. 11 and Fig. 12 a) corresponds to either red, green or both channels in their ON state (under front irradiation). Observing both figures a threshold line $\left(\mathrm{T}_{\mathrm{Fa}}\right)$ can be drawn above which, any result is the function $\mathrm{D}$ OR P, i.e. $\{\{01\},\{10\},\{11\}\}$.

The Exclusive OR function, XOR, between two variables $\{\mathrm{DP}\}$ is True when only one of them holds the True value, i.e. $\{\{01\},\{10\}\}$. It excludes the $\{11\}$ from the OR. It can also be defined as the difference operation, which is True, when the variables hold a value which is different from the other. Observing the scatter graph of Fig. 12 a) it is possible to confine the $\{\{01\},\{10\}\}$ values between the two threshold lines $\mathrm{T}_{\mathrm{Fa}}$ and $\mathrm{T}_{\mathrm{Fb}}$.

\section{Memory Effect}

The p'inpin device has been used throughout this work as a stateless component which means that its output is only dependent of its inputs regardless of the outcome history of past inputs or outputs. This however does not hold when the outputs are observed as a sequence of inputs. The identification of the input sequence, using a classification function with the output of the pi'npin, shows that past sequences do influence the output of the device which does in fact hold a memory of the illumination beforehand. Although this is a difficult task for signal identification it is necessary to understand this phenomenon to ease the identification task and also to use it as an advantage by constructing a volatile memory with the pi'npin device.

\section{Paradigm Change}

The observations made to indicate that there is a memory function that characteriz- 
es the pi'npin device started when the readings of the dark signal did not remain constant between experiments. Initially it was assumed that there was an influence of noise from the surrounding equipment and other devices. Several modifications were gradually made to the experimental setup to reduce the influence of the noise sources.

To understand the start condition of the pi'npin device and its influence over the output, changes were made to the software of the user interface and that of the microcontroller that drives the LEDs, to allow static readings [24, 25]. The selection of a start condition, programmable delays and the possibility of short sequences, all time controlled and with adequate triggering for the oscilloscope, allowed the study that is presented in this section.

In all experiments till this point the optical bias was used as a steady lighting, namely in the digital light logical functions and WDM [18, 21, 26]. This optical bias paradigm has to be changed, and seen as two light Control signals; the Back and Front Control signals, to use the sensor as a volatile memory [31]. On this section, the Front and Back lighting are no longer steady state, but impulses.

\section{Conceptual Approach}

The experimental setup with the relative positioning of the LEDs to the sensor is shown in Fig. 13 a) along with the equipment. The LED currents and their timing patterns are controlled by PiscaLed system [24, 25].
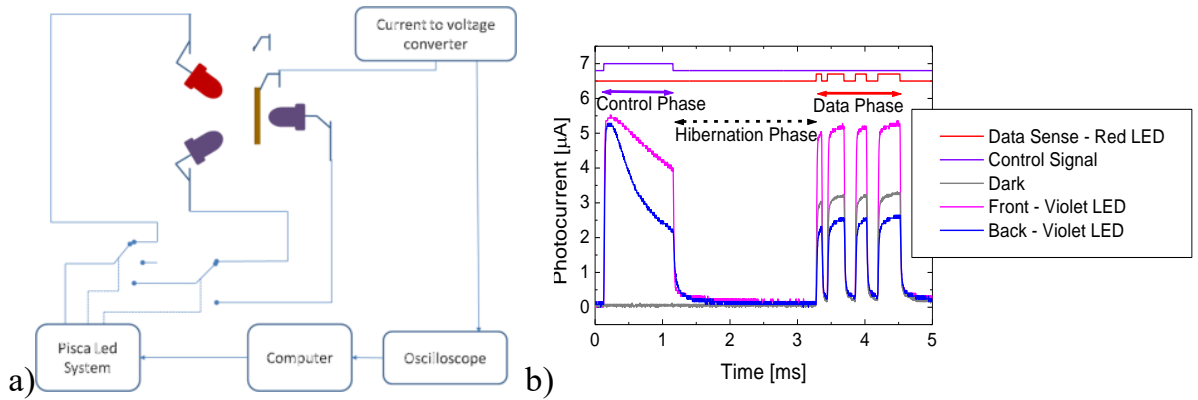

Fig. 13. a) Experimental Setup showing D (Data), H (Hibernation) and C (Control) phases as switches. b) Outputs represented on same plot by three different conditions: Dark, Front Control and Back Control.

There is a sequential operation with three phases to use the sensor as a volatile memory: Control, Hibernation and Data phase. Control phase is when only the Front Control and or the Back Control signals lit the sensor. The Hibernation phase has no illumination over the sensor. Data phase when the sensor is illuminated with Data Sense signal. Phases are represented in Fig. 13 as switches a), and as a timeline b).

Data Sense signal Phase duration: to study the decay of the stored value through time, an experiment was set in which the Data Sense signal used in the previous experiments is repeated cyclically with a $0.5 \mathrm{~ms}$ interval. This is shown in Fig. 14, where the signals at the top guide the eyes. Presented in Fig. 14, are the results of the applied Back and Front Control signals, each with duration of $3 \mathrm{~ms}$ followed by the 
Hibernation phase lasting $3 \mathrm{~ms}$. The Data Sense signal sequence is equivalent to 16 cycles at a frequency of $6000 \mathrm{~Hz}$. These 16 cycles are then repeated.

Results show that there is amplitude decay in time of the output Data Sense signal despite the Control signal is either Front or Back. The decay rate, were it linear, is roughly twice higher in the Front Control signal $(-83 \mu \mathrm{A} / \mathrm{s})$ than in the Back Control signal $(-46 \mu \mathrm{A} / \mathrm{s})$ which means that they instead of crossing will maintain an amplitude equivalent to the Dark reference signal.

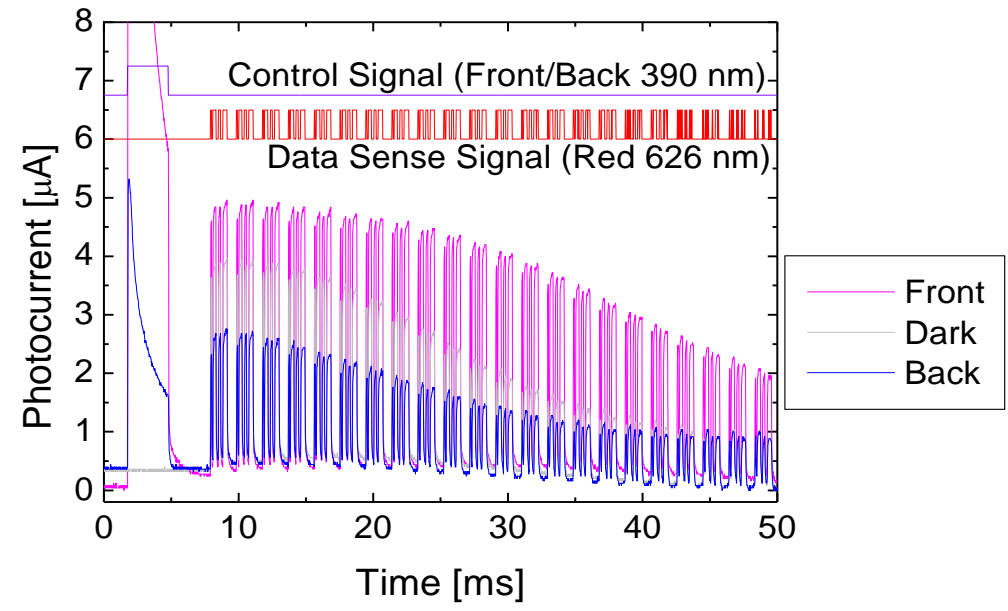

Fig. 14. Back and Front Control signals followed by Data Sense with a repeated cycle pattern.

Furthermore, the decayed amplitude of the Data Sense due to the Front Control will eventually be less than the highest amplitude of the Data Sense due to the Back Control. Observing Fig. 14 that would occur around $25 \mathrm{~ms}$; this determines the volatile memory hold time due to the data sense signal profile.

\section{Conclusion}

The p'inpin device has proven to be versatile and with several applications in which it was used: multiplexer, logical gates and a volatile memory. This flexibility is its main advantage.

By experimental observation the simultaneous incidence of the front and back optical illumination is equivalent to a front bias illumination. This characteristic could be used by having the back surface always illuminated and using the front bias when convenient which could speed up the acquisition process.

The sensor illumination has always flooded the whole surface. Studying the use of masks to allow only certain parts of the sensor being used by spatially distributing the input wavelengths over the surface can produce curious results.

The photocurrent varies linearly with the flux intensity of the input signals. By using analogue signals a "light transistor" can be built using the background illumination as a constant amplification setting. 
The possibility to change the pi'npin heterostructures by depositing more pins and enabling their junctions to be accessible by optical transparent contacts will definitely improve the research of this simple photosensitive device. The ideal device would be one that could also emit light; with optical inputs and outputs it would effectively be considered as an optical gate. The demultiplexer would then emit in separate channels, the logical functions would work as gates that could be connected as circuits including the memory component.

Due to the low throughput of 12000 bps of each channel the pi'npin sensor can be used in audio and signalling applications.

Acknowledgments. Work supported by FCT, CTS multi annual funding, through PIDDAC Programs PTDC/EEA-ELC/111854/2009, PTDC/EEA-ELC/120539/2010.

\section{References}

1 M. Vieira, A. Fantoni, P. Louro, M. Fernandes, R. Schwarz, G. Lavareda, and C. N. Carvalho, "Self-biasing effect in colour sensitive photodiodes based on double p-i-n a-SiC:H heterojunctions," Vacuum, vol. 82, no. 12, pp. 1512-1516, Aug. 2008.

2 M. Vieira, M. Fernandes, P. Louro, M. A. Vieira, M. Barata, and A. Fantoni, "Multilayered a-SiC:H device for Wavelength-Division (de)Multiplexing applications in the visible spectrum," MRS Proc., vol. 1066, pp. 1066-A08-1, Feb. 2011

3 The Royal Academy of Engineering, "Smart infrastructure: the future."

4 M. Vieira, P. Louro, M. Fernandes, M. A. Vieira, Q. Torre, and M. Caparica, "Three Transducers Embedded into One Single SiC Photodetector : LSP Direct Image Sensor, Optical Amplifier and Demux Device," in Advances in Photodiodes, vol. March Ch19, G. F. D. Betta, Ed. Intech, 2011, pp. 403-426.

5 G. De Cesare, F. Irrera, F. Lemmi, F. Palma, and M. Tucci, "a-Si:H/a-SiC:H Heterostructure for Bias-Controlled Photodetectors," MRS Proc., vol. 336, p. 885, Feb. 2011.

6 M. A. Vieira, "Three Transducers for One Photodetector: essays for optical communications," FCT-UNL Universidade Nova de Lisboa, 2012.

7 V. Silva, "PiscaLed - User's Manual," GIAMOS - ISEL/IPL, 2013.

8 V. Silva, "PiscaLed - Technical Manual," GIAMOS - ISEL/IPL, 2013.

9 V. Silva, "HexaLed- User's Manual," GIAMOS - ISEL/IPL, 2016.

10 V. Silva, "IndicaLed," GIAMOS - ISEL/IPL, 2015.

11 V. Silva, "Papagaio," GIAMOS - ISEL/IPL, p. 6, 2015.

1] V. Silva, "Descodifica," GIAMOS - ISEL/IPL, 2013.

13 ATMEL, "Manchester Coding Basics: Application Note," 2009.

14 D. Choi, "Report: Parallel Scrambling Techniques for Digital Multiplexers," AT\&T Tech. J., vol. 65, no. 5, pp. 123-136, Sep. 1986.

15 V. Silva, M. Barata, P. Louro, M. A. Vieira, and M. Vieira, "Five channel WDM communication using a single a:SiC-H double pin photo device," Appl. Surf. Sci., Nov. 2015.

16 V. Silva, "Reconfigurable photonic logic architecture," UNL - Universidade Nova de Lisboa, 2015 .

17 G. Boole, "The mathematical analysis of Logic," 1847.

18 H. Nishimura, "Boolean valued decomposition theory of states," Publ. Res. Inst. Math. Sci., vol. 21, no. 5, pp. 1051-1058, 1985.

19 A. Tanenbaum and T. Austin, Structured computer organization, 6th ed. Pearson Education International, 2013.

20 Millman, Microelctronics. Mcgraw Hill. 
21 V. Silva, M. A. Vieira, P. Louro, and M. Vieira, "Optoelectronic digital capture device based on $\mathrm{Si} / \mathrm{C}$ multilayer heterostuctures," in Technological Innovation for the Internet of Things, Springer Berlin Heidelberg, 2013, pp. 555-562.

22 J. B. Moore and K. T. Tan, "Majority Logic Coding and its Multinomial Representation," no. $2,1975$.

23 W. Stallings, Computer Organization And Architecture Designing For Performance., 8th ed. Prentice Hall.

24 V. Silva, M. A. Vieira, P. Louro, M. Barata, and M. Vieira, "Simple and complex logical functions in a $\mathrm{SiC}$ tandem device," in Technological Innovation for Collective Awareness Systems, 2014, pp. 592-601.

25 M. Vieira, M. A. Vieira, V. Silva, P. Louro, and J. Costa, "SiC monolithically integrated wavelength selector with 4 channels," MRS Proc., vol. 1536, pp. 79-84, Jun. 2013.

26 V. Silva, M. A. Vieira, M. Vieira, P. Louro, A. Fantoni, and M. Barata, "Logic functions based on optical bias controlled SiC tandem devices," Phys. Status Solidi, vol. 11, no. 2, pp. 211-216, Feb. 2014

27 V. Silva, M. Vieira, P. Louro, and M. Barata, "Logical functions in a tandem SiC device," Microelectron. Eng., vol. 126, pp. 79-83, Jun. 2014.

28 W. Navidi, Statistics - for Engineers and Scientists, 3rd ed. McGraw-Hill Professional, 2010.

29 V. Silva, M. A. Vieira, P. Louro, M. Barata, and M. Vieira, “AND, OR, NOT Logical Functions in a SiC Tandem Device," Procedia Technol., vol. 17, pp. 557-565, 2014.

30 G. C. Valley, "Photonic analog-to-digital converters.," Opt. Express, vol. 15, no. 5, pp. 1955-1982, 2007.

31 P. Louro, M. Vieira, J. Costa, M. A. Vieira, M. Fernandes, A. Fantoni, and M. Barata, "aSiC:H Based Devices as Optical Demultiplexers," MRS Proc., vol. 1246, pp. 1246-B07-7, Feb. 2011. 